\title{
Desarrollo de competencias en educación económica y financiera para la toma de decisiones informadas del ciudadano común
}

\author{
Development of competences in economic and financial education for informed \\ decision making of the common citizen
}

\section{Desenvolvimento de competências em educação econômico-financeira para tomada de decisão informada do cidadão comum}

\author{
Sonia Valbuena- Duarte ${ }^{\mathrm{a}^{*}} \mid$ Karina Andrea Marín-Tapia ${ }^{\mathrm{b}}$ |Angely Paola De la Hoz \\ a http://orcid.org/0000-0003-3667-1087 \\ ${ }^{\mathrm{b}}$ http://orcid.org/0000-0002-9722-8663 \\ c http://orcid.org/0000-0002-6920-2239 \\ Universidad del Atlántico, Colombia
}

- Fecha de recepción: 2019-II-14

- Fecha concepto de evaluación: 2019-1/-26

- Fecha de aprobación: 2019-12-04 http://dx.doi.org/I0.22335/rlct.v|2il.II 03

\begin{abstract}
Para citar este artículo / To reference this article / Para citar este artigo: ValbuenaDuarte, S., Marín-Tapia, K. A., \& De la Hoz, A. P. (2020). Desarrollo de competencias en educación económica y financiera para la toma de decisiones informadas del ciudadano común. Revista Logos Ciencia \& Tecnología, I2(I), 95-I09. http://dx.doi.org/I0.22335/rlct.v I 2i I.I I 03
\end{abstract}

\section{RESUMEN}

Esta investigación tuvo como objetivo desarrollar en docentes competencias en educación económica y financiera (EEF), en busca de que estos en su práctica pedagógica formen a los jóvenes como ciudadanos que tomen decisiones informadas respecto de temas económicos y financieros e impacten sus proyectos de vida con calidad y sostenibilidad. Se hizo uso de la metodología de la investigaciónacción, se realizó revisión documental, observación, diario de campo y encuestas a docentes, lo cual dejó en evidencia la ausencia de estas competencias. Como producto de investigación, se plantean orientaciones al docente para la articulación de este saber en la toma de decisiones informadas, en consideración a los lineamientos del Ministerio de Educación Nacional (MinEducación) sobre EEF,y se logró impactar a través de un programa de formación docente a 13 instituciones del departamento del Atlántico, públicas y privadas.

Palabras clave: desarrollo de las habilidades, educación del consumidor, educación formal, formación de docentes, toma de decisiones 


\begin{abstract}
SUMMARY
This research aimed to develop competences in economic and financial education (EEF) in teachers, in order for them, in their pedagogical practice, to train young people to become citizens who make informed decisions regarding economic and financial issues and impact their life projects with quality and sustainability The action-research methodology was used, while carrying out the revision of documents, field dairies and observations; teacher surveys were also carried out, all evidencing the absence of these competences. As a result, teacher guidelines are proposed for the articulation of making informed decisions, taking into consideration the guidelines of the Ministry of Education (MinEducación) in relation to EEF, and through a teacher training program, 13 public and private institutions in the department of Atlántico were impacted.
\end{abstract}

Keywords: skills development, consumer education, formal education, teacher training, decision making

\title{
SUMÁRIO
}

Esta pesquisa teve como objetivo desenvolver nos professores competências em educação econômica e financeira (EEF), a fim de que estes, em sua prática pedagógica, formem jovens como cidadãos que tomam decisões informadas sobre questões econômicas e financeiras impactando seus projetos de vida com qualidade e sustentabilidade. Utilizou-se a metodologia de pesquisa-ação, revisão documental, observação, pesquisa de campo e pesquisas com professores, o que evidenciou a ausência dessas competências. Como objeto de pesquisa, são propostas diretrizes para os professores para a articulação desse conhecimento na tomada de decisões informadas, considerando as diretrizes do Ministério da Educação Nacional (Ministério da Educação) sobre EEF, e o impacto foi alcançado por meio de um programa de formação docente a 13 instituições públicas e privadas do departamento do Atlántico.

Palavras-chave: desenvolvimento de habilidades, educação do consumidor, educação formal, formação de professores, tomada de decisão

Los individuos a diario se proveen de bienes y servicios, lo que hace necesario poseer capacidades y habilidades para el manejo adecuado de situaciones financieras, de tal forma que no afecten su estabilidad económica. Hoy día, la educación económica y financiera (EEF) es considerada importante para las personas, puesto que a través de estas pueden aprovechar los servicios y productos financieros, aprender a administrar adecuadamente sus ingresos y gastos, tomar mejores decisiones, evitar altos niveles de endeudamiento y mejorar la calidad de vida. Por su parte, se ha evidenciado que en la población colombiana las deudas de consumo y de vivienda alcanzan hoy alrededor del $20 \%$ del producto interno bruto (PIB) del país, lo que traduce los altos niveles de endeudamiento presentes en la ciudadanía (Semana, 2018). Una posible causa de este panorama es la ausencia de competencias básicas en los ciudadanos en el momento de tomar decisiones económicas y financieras.

Desde posturas como las de Murillo y Cáceres (2013), la informática dispone de información financiera al ciu- dadano común para tomar buenas decisiones, de modo que esta se convierte en insumo fundamental para tomar decisiones informadas.

Por otro lado, en América Latina la EEF se ha convertido en eje fundamental para sectores educativos y entes bancarios que trabajan para impulsar programas que promuevan la educación financiera en la comunidad, brindando conocimientos de temas económicos y financieros para fomentar una cultura financiera en los ciudadanos. Reyes (2002) mencionan que los bancos centrales de América Latina vienen desarrollando programas de EEF con la intención de mantener una cierta comunicación con la población que, junto con el Ministerio de Hacienda y el Ministerio de Educación, poseen la potestad de proveer la infraestructura y los recursos para difundir la EEF.

Los bancos centrales en el mundo recientemente vienen cumpliendo una función cada vez más extensa, desarrollando programas de EEF que fomenten la cultura financiera como eje principal para los países, dado que elevan 
la efectividad de la política monetaria y promueven tanto la aceptación de las acciones de los bancos centrales como el reconocimiento público de la institución. En particular, para el banco central de Colombia adoptar una cultura financiera contribuye a suavizar el funcionamiento de los mercados financiero, puesto que los consumidores más educados $y$ mejor informados toman mejores decisiones financieras a lo largo de su vida, y así cuidan su bolsillo y, en general, el de sus allegados, por lo cual se reducen probabilidades de crisis económica y la adquisición de una mayor conciencia (Ministerio de Hacienda, 20I4).

En este sentido, Ley $1328 / 2009$, de 15 de julio establece que las entidades del sector financiero vigiladas por la Superintendencia Financiera de Colombia (SFC) tienen la potestad de desarrollar programas de EEF para promover la inclusión de una cultura financiera en los ciudadanos. Es decir, propiciar el desarrollo de capacidades financieras que permitan a las personas actuar de manera adecuada en cuanto a la toma de decisiones informadas en situaciones que se les presente en su vida diaria.

Ahora bien, para llegar a ser un ciudadano que toma decisiones, lo regular es que el colegio forme y potencie esta competencia. Gamboa, Hernandez y Avendaño (2018) consideran que para formar a los ciudadanos económica y financieramente este saber debe ser considerado en las escuelas incluyendo los procesos formativos que conlleven el desarrollo de competencias y capacidades asociadas a la toma de decisiones en cuanto a la administración de los recursos económicos.

A nivel mundial, países como los Estados Unidos, Brasil y el Reino Unido han sido reconocidos como territorios líderes en asuntos como regulación, desarrollo de programas y puesta en marcha de proyectos e iniciativas para promover la EEF entre sus ciudadanos, y crear en los últimos años estrategias para desarrollar competencias y habilidades en EEF desde las instituciones educativas en sus planes de estudios de manera transversal.

Con respecto a programas de EEF, se destaca una iniciativa elaborada por Sepúlveda (2014) que establece la necesidad de incorporar la EEF como una herramienta en las escuelas y la sociedad. Con la revisión de estrategias didácticas y metodológicas de la enseñanza, el desarroIlo, los ejes temáticos, las competencias específicas, los resultados de aprendizaje y las actividades, se logra brindar este material que describe el modelo de formación inicial que deben optar los docentes para la promoción de la alfabetización de la EEF en el ámbito escolar, a través de actividades pedagógicas, de tal manera que permita desarrollar competencias en el momento de incorporar la alfabetización de EEF en niños y jóvenes en sus clases.

En Colombia, se destacan estudios como el realizado por Cristancho, Cruz, Echeverry, Rojas y Urrego (2017), los cuales identifican la relación que existe entre la implementación de una asignatura enfocada en educación financiera dentro del plan de estudios y el nivel de conocimientos financieros de los estudiantes de educación media en el Colegio Pablo Neruda de la localidad de Fontibón, para lo cual emplearon la metodología de nuevas estrategias mediante un propuesta curricular que permita implementar las orientaciones dadas por el Ministerio de Educación Nacional (MinEducación) para la EEF. De esta manera, brindan una guía de trabajo para incluir en el currículo del colegio la educación financiera como una asignatura para estudiantes de media vocacional.

En el orden nacional, el programa pionero de educación financiera se denominó Finanzas para el Cambio en 2005, el cual pretendía insertar paulatinamente en la educación básica y media conceptos de esta área del saber cómo parte de los currículos escolares de las instituciones educativas del país. Este programa fue desarrollado por la Fundación Dividendo por Colombia, la Fundación Corona y Citibank-Colombia, mediante alianzas estratégicas con las secretarías de Educación de Bogotá, Cartagena y Medellín (Asociacion Bancaria y de Entidades Financieras de Colombia [Asobancaria], 2016).

La segunda gran iniciativa fue el Programa de Educación Financiera, apoyado por la Fundación Bancolombia en 2009. Durante sus cuatro años de duración, este programa atendió a 175 instituciones educativas en 39 entidades territoriales, y se reportó capacitación a más de 4000 docentes, más de 13000 padres de familia y 128249 estudiantes (Asobancaria, 20I6).

En atención a las orientaciones del MinEducación, se implementaron iniciativas a nivel local, las cuales brindaron herramientas básicas y permitieron a los ciudadanos, en especial a las familias de Barranquilla, tomar decisiones financieras acertadas. Estas hicieron el uso adecuado de los recursos de su hogar y asumieron los nuevos retos que afrontarán en sus residencias ayudando a desarrollar 
esas habilidades para la planeación y administración de sus recursos (El Heraldo, 20I3).

Otra de las iniciativas propuesta por el MinEducación y entidades del sector financiero en 2010 consistió en implementar en Colombia la Estrategia Nacional de Educación Económica y Financiera (ENEEF) que facilite mejorar la manera en la que se ofrece la EEF a la población del país en las distintas etapas de la vida (Centro de Investigación y Estudios en Economía Financiera [CIEEF], 2017).

Esta estrategia se desarrolló bajo los principios y estándares ofrecidos por organismos internacionales, tales como la Organización para la Cooperación y el Desarrollo Económico (OCDE), la cual coordina sus políticas económicas y sociales, y a través de programas como las pruebas PISA (Programme for International Student Assessment) evalúa cómo los sistemas educativos preparan a sus estudiantes para que apliquen sus conocimientos y habilidades en tareas que son relevantes para su vida actual y futura. Del mismo modo, por medio de tres competencias (científica, matemática y lectora), evalúa las habilidades de los estudiantes para analizar y resolver problemas, para manejar información y enfrentar situaciones que se presenten en la vida adulta (OCDE, 2007).

Estas pruebas van dirigida a jóvenes colombianos en edad promedio de 15 años. La OCDE aplica este examen estandarizado cada tres años, desde 2000, y en cada año de su aplicación profundiza en una de las tres competencias a evaluar. Dentro de la competencia matemática, se encuentra la $E E F$, la cual busca desarrollar en los niños, niñas $y$ adolescentes los conocimientos y las habilidades necesarios para la toma de decisiones en temas económicos y financieros presentes en su vida escolar, familiar y social (OCDE, 2005).

Como parte del proceso de adhesión, Colombia en tanto país invitado sometió a sus estudiantes a estas pruebas por primera vez en 2006 , y solo hasta 2018 llega a ser país miembro de la OCDE. Por otro lado, según PISA 2012 el $70 \%$ de los 10 países con mayores puntajes en conocimientos financieros han integrado la educación financiera en sus programas escolares o universitarios. Esto se evidencia en países como Brasil, Chile y Singapur, dado que cuentan con una estrategia nacional de educación financiera establecida, que ha fortalecido el desarrollo de estas competencias en sus ciudadanos (Asobancaria, 2016).
Por su parte, Colombia no ha sido uno de los países con mejores puntajes en estas pruebas, puesto que desde su primera participación como país invitado ha obtenido muy bajos puntajes. En 2012, las pruebas PISA profundizaron en la competencia matemática; ese año el puntaje promedio de Colombia en esta competencia fue 376 puntos, por debajo de la media de la OCDE (494 puntos ese año), y ocupó el puesto 62 de 65 países/economías evaluadas. Cabe resaltar que ocupó el último lugar en lo referente a resolución de problemas y EEF, lo cual muestra que más del $50 \%$ de los estudiantes está por debajo de la línea base (media OCDE). Los resultados de estas pruebas reflejan la falta de EEF en los jóvenes colombianos, dado que en muchos casos los estudiantes tuvieron dificultad, por ejemplo, en distinguir la diferencia entre las necesidades económicas y los deseos. Lo máximo que pudieron hacer fue tomar "decisiones sencillas sobre gastos rutinarios, reconocer la función de una factura, y realizar operaciones numéricas básicas (sumas, restas o multiplicaciones) (OCDE, 20I4).

En este sentido, el MinEducación y Asobancaria, con el propósito de consolidar una educación de calidad, firman un convenio para la implementación del programa de EEF en los establecimientos educativos del país. Para así responder mejor las pruebas de 2015, el MinEducación realizó en 2014 un ejercicio piloto para la implementación de la EEF en 120 instituciones de cinco regiones del país, en zonas rurales y urbanas, ofreciendo el material Mi plan, mi vida y mi futuro: orientaciones pedagógicas para la educación económica y financiera donde el MinEducación redefine la EEF como un proyecto pedagógico transversal articulado al desarrollo de competencias en las áreas básicas y ciudadanas, promoviendo programas de formación y capacitación docente $y$ ofreciendo manuales y fichas didácticas diseñadas para la enseñanza (MinEducación , 20l4).

A pesar de estas decisiones en busca de mejores resultados, Colombia en las pruebas PISA 2015 solo subió 14 puntos en comparación con las pruebas PISA 2012 y ocupó el puesto 59 de 72 países/economías participantes, por lo cual permaneció en niveles de desempeño bajos en EEF, matemáticas y las otras áreas en general. Frente a los resultados de estos estudiantes y las acciones identificadas en docentes de Matemáticas en 13 instituciones educativas del departamento del Atlántico, esto permite inferir que desde los colegios los docentes no tenían ninguna formación en EEF y además desconocían los lineamientos de EEF propuestos por el MinEducación. 
Puesto que en los docentes de Matemáticas y de Ciencias Sociales recae la responsabilidad de formar en competencias y desarrollar habilidades en EEF en sus estudiantes, hay que resaltar que todas las demás áreas específicas son relevantes para el desarrollo de estas competencias, dado que los problemas que se generan en los contextos son de comunidades. Así, se puede asumir desde las prácticas de las ciencias naturales, dados los cambios vividos por los países desarrollados, donde se considera a las fuentes naturales como los principales orígenes de valor que tienen los países; de esta manera, se puede ir comprendiendo que la educación financiera se puede ver como un componente globalizante en la escuela que brinda posibilidades reales de conocimiento a las personas que se encuentran en formación (Mogollón-Guerrero, Molano-Castro, \& Mesa-Jiménez, 2017).

Queda claro que la formación del docente desempeña un papel fundamental en los aprendizajes conceptuales de economía y finanzas en los estudiantes. Sin embargo, resulta preocupante, ya que los docentes de Matemáticas y Ciencias Sociales en particular no cuentan con las capacidades necesarias para impartir estos conocimientos. Y no se evidencia que en su formación en educación superior hayan tenido esta formación desde su plan de estudio, como tampoco hay hallazgos de formación ni actualizaciones en este sentido en su vida profesional y de desempeño.

En atención a lo anterior, este artículo pretende desarrollar tales competencias en docentes de Matemáticas y Ciencias Sociales, de tal forma que en su práctica pedagógica logren potenciar dichas competencias en sus estudiantes. Para esto, se diseña e implementa un programa de formación docente dirigido a los que se les ha atribuido reglamentariamente esta responsabilidad, Matemáticas y Ciencias Sociales. Dicho programa se ejecutó con docentes de 13 instituciones educativas públicas y privadas del departamento del Atlántico (Colombia), reconociendo que la EEF se plantea como un proyecto pedagógico de carácter transversal.

\section{Desarrollo de programas, contenidos y} herramientas de EEF en Colombia

Con la intención de promover la EEF en Colombia, Asobancaria (2016) busca concientizar a la población de la importancia de educarse financieramente brindando herramientas para mejorar los conocimientos y hábitos de las personas a la hora de manejar sus finanzas personales y familiares; en este sentido, han desarrollado programas de educación financiera acompañados de una estrategia digital. Así, en 2009, pone en marcha su primer portal de educación financiera $A B C$ mi Casa, una herramienta on-line con toda la información necesaria para que las personas puedan tomar decisiones adecuadas en lo relacionado con el crédito hipotecario y con el proceso de compra y mantenimiento de la vivienda. En 20I2, lanza el portal Cuadre su Bolsillo para informar sobre los asuntos más relevantes en educación financiera, sitio web que contenía consejos útiles e información práctica sobre asuntos clave para el adecuado manejo de las finanzas personales y del hogar. Puesto que en 2015 este portal migra a un nuevo portal, Saber Más, Ser Más (www.sabermassermas.com), el cual se encuentra disponible hasta la fecha de hoy, cabe resaltar que en $2016 \mathrm{ABC}$ mi Casa se integra a este nuevo sitio e incluye funcionalidades multimedia de adaptarse al acceso desde distintos dispositivos tales como tabletas - móviles, donde Asobancaria brinda herramientas que permitan tener la información necesaria para la toma de decisiones informadas para fortalecer la inclusión y el acceso a los servicios financieros y el consumo responsable en los ciudadanos colombianos.

De la misma manera, y con intención de promover la EEF, el Estado colombiano toma principios, prácticas y experiencias de países que han sido reconocidos como territorios líderes en regulación, desarrollo de programas y puesta en marcha de proyectos e iniciativas para promover la EEF entre sus ciudadanos, y fueron considerados para la elaboración de la ENEEF, desde la CIEEF (2017). Evidentemente, Colombia ha realizado intentos por brindar una educacion financiera que se pueda ver reflejada en una sociedad en el futuro, así como se establece en el documento Mi plan, mi vida, mi futuro: orientaciones pedagógicas para la educación económica y financiera del MinEducación (20I4), que brinda una ayuda para la implementación de la EEF en las escuelas.

Así es como, teniendo como propósito educar a la ciudadanía financiera y económicamente, la CIEEF a través de la ENEEF recalca el plan de acción en el que a partir de etapas plasma recomendaciones al documento "Mi plan, mi vida, mi futuro: orientaciones pedagógicas para la educación económica y financiera con énfasis en la educación formal y en dirigir y enviar materiales de apoyo para los colegios 
y docentes, ocupándose de la EEF también del adulto a través de la página web (www.pesospensados.gov.co). En síntesis, los programas de EEF centraron su atención en la complejidad de los mercados y productos financieros, en la atribución de importantes ventajas individuales y sociales a la extensión de la cultura financiera entre la ciudadanía (Domínguez, 2015).

Pese a todo el esfuerzo y la creación de planes desde hace una década, se evidencia desconocimiento de lineamientos y políticas en los profesores muestra objeto de estudio ( 3 colegios del departamento del Atlántico en Colombia). Sus planeaciones de áreas curriculares no reflejan conocimiento alguno de estas intenciones, con ausencia clara de estas competencias en los docentes, que a nivel escolar son quienes las deben crear, fortalecer y potenciar en los estudiantes, lo que señala de igual manera ausencia de competencias en EEF en los jóvenes estudiantes.

\section{Programas de formación y capacitación} docente

Siendo manifiesta la necesidad de formar al docente en competencias en EEF, el programa de formación docente se concibe como el conjunto de estrategias, procesos y acciones de gestión académica, en busca de actualizar y profundizar conocimientos, habilidades y destrezas para desarrollar la docencia, la investigación y la extensión con calidad, la innovación y el alto sentido de compromiso con las necesidades de la región y el país en el caso de la EEF.Así, para la conformación del programa de formación, se hizo uso de pautas del Instituto Superior de Educación Rural (ISER, 20I5).

Según Reyes (2002), un programa de formación docente debe tener una dimensión real dentro del contexto económico, social y político de un país, y considerar que la educación es un servicio público y de naturaleza social. Por su parte, para Taborga y Hanel (1992), los programas de formación deben ser concretos, delimitados y propositivos, evitar que sean muy discursivos y teóricos, y darles operatividad mediante la aplicación de criterios que consideren el grado de importancia, pertinencia y factibilidad. De esta manera, se resalta la importancia de articular acciones rutinarias e innovadoras en un programa de formación docente, ya que atribuyen a la administración educativa y la planeación el desarrollo de habilidades que requieren las rutinas cotidianas del ejercicio docente, estimulando la producción de capacidades y competencias para ejercer diversas actividades que correspondan a una amplia visión de trabajos y responsabilidades que se cumplen en las instituciones educativas.

Dado que el programa de formación docente implicó plantear un rediseño curricular en las áreas disciplinares implicadas, en busca de que los conocimientos en EEF se mostraran de manera integrada, se tomaron autores como Godino (2004) y los seis principios para el desarrollo de diseños curriculares. Estos orientaron la planificación de unidades didácticas, la selección de materiales, el diseño de evaluaciones, las decisiones instruccionales en las clases y el establecimiento de programas de apoyo para el desarrollo profesional de los docentes, todas estas orientadas a la enseñanza y aprendizaje:

Equidad, la excelencia en la educación requiere equidad, unas altas expectativas y fuerte apoyo para todos los estudiantes.

Currículo, coherente, centrado en saberes importantes y bien articulados por niveles.

Enseñanza, efectiva con la comprensión de lo que los estudiantes conocen y necesitan aprender.

Aprendizaje, con comprensión, construyendo activamente el nuevo conocimiento a partir de la experiencia y el conocimiento previo.

Evaluación, apoyando el aprendizaje de saberes importantes y proporcionando información útil tanto a los profesores como a los estudiantes del proceso.

Tecnología, estimulando el aprendizaje de los estudiantes.

En el diseño curricular, se establecen tres tipos de contenidos (conceptual, procedimental y actitudes), y siguiendo a Godino (2004), el aprendizaje se brinda de manera intencional por lo cual favorece situaciones de enseñanza, desarrolla valores, normas y actitudes oportunas. De esta manera, en la articulación de la EEF se requiere:

- Conocer el proyecto transversal de EEF y todo lo que encierra sus ejes temáticos para articularlo a las áreas de conocimientos.

- Trabajar en equipo con los docentes de las demás áreas disciplinares.

- Diseñar situaciones problemas tomadas del contexto del estudiante y partir de situaciones de aprendizaje significativo articuladas a la EEF para incentivar la toma de decisiones informadas. 
- Facilitar la reflexión y aplicación práctica de los conceptos en situaciones económicas y financieras de escenarios vinculados a las experiencias cotidianas de los estudiantes.

- Resaltar a los estudiantes la importancia del sistema económico y financiero a partir de la valoración de los recursos que poseen e identifiquen los demás de su entorno que por ser escasos requieren buen uso y cuidado para favorecer su conservación y aprovechamiento sostenible.

- Crear ambientes de aprendizaje que transciendan el material didáctico y el recurso físico del aula que fomenten el aprendizaje autónomo y cooperativo en los estudiantes en EEF, lo cual tendrá como ganancia adicional el desarrollo de pensamiento crítico ciudadano (Hernández, Londoño, \& Silva, 2019), ayudará a generar conocimiento, desarrollará pensamiento para solucionar problemas en la vida diaria, contribuirá a formar voluntad y adquirir autonomía en los actos del joven, y aportará en la transformación de la realidad.

- Diseñar estrategias evaluativas en atención a un enfoque integral, flexible y formativo, asimismo elaborar instrumentos de evaluación del aprendizaje según los estándares básicos de competencias delineados por el Min Educación y las orientaciones que brinda sobre EEF.

- Promover la participación de la familia en el proceso de formación en EEF de los estudiantes.

Las actividades de la propuesta de este trabajo de investigación siguen las ideas de Godino (2004), quien centra su enseñanza en que el aprendizaje es siempre el producto de la actividad, y si esta se reduce, por ejemplo, a la resolución repetitiva de ejercicios para aplicar ciertas fórmulas, esto es lo que se aprende y lo que queda en los alumnos. Por esta razón, las actividades deben superar el aprendizaje pasivo, con el uso de materiales, problemas contextualizados, grupos de trabajo, uso de diferentes representaciones, la contextualización de contenidos, etc.

\section{Evaluación del programa en EEF}

Es importante considerar en el programa de EEF la evaluación interna del proceso de formación en los docentes como parte de un conjunto de criterios de calidad orientados por principios de consistencia y credibilidad. Siendo la evaluación un ejercicio esencial para ayudar a perfeccionar los diferentes aspectos de la operación del programa en EEF para implementar un proceso de mejoramiento continuo, se toma del Programa Institucional de Formacion Docente (Universidad de Colima, 2012) lo continuo, sistemático y multidimensional del proceso, del cual se recoge información relevante, válida y fiable para tomar decisiones sobre el valor de este. En la evaluación, se sigue el modelo propuesto por Stufflebeam y Shinkfield (1987), denominado CIPP (Context, input, Process, Product), cuyo propósito es recolectar información para tomar decisiones en el programa, en el cual se integran estas cuatro dimensiones, en cinco fases, como se muestra en la Figura I.

Figura I. Fases de evaluación del programa de formación docente

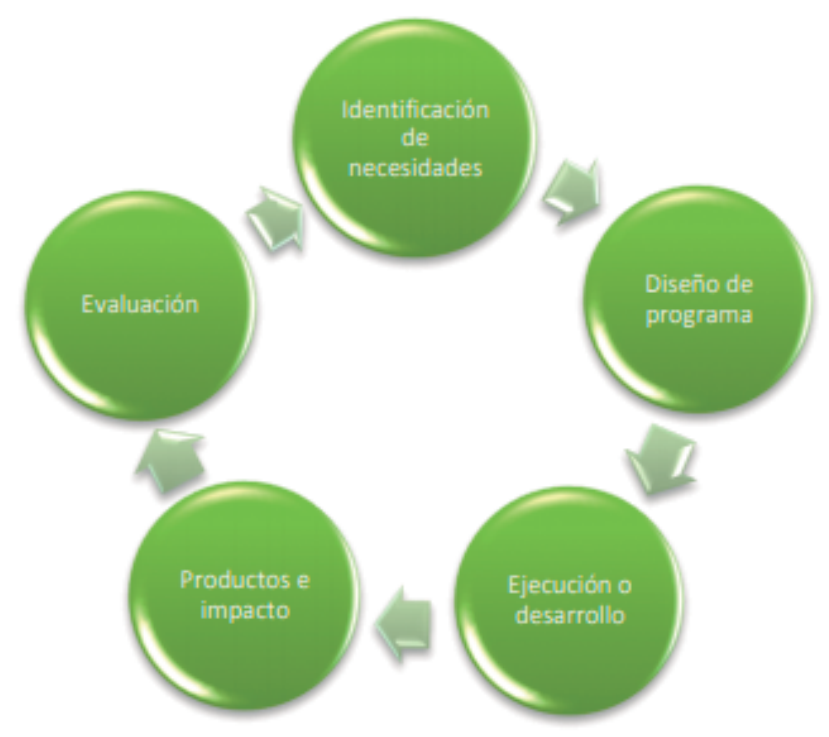

Fuente: Universidad de Colima (20I2).

\section{Método}

Esta investigación sigue una metodología de carácter cualitativo con un diseño desde la investigación-acción (IA) (Creswell, 2012; Hernández, Fernández, \& Baptista, 20l4), la cual comprende y resuelve problemas específicos de un grupo perteneciente a un mismo entorno, quienes transforman la realidad educativa, donde los participantes toman conciencia de su papel en ese proceso de transformación.

De acuerdo con lo anterior, siguiendo la metodología IA, se establecen cinco etapas: la primera etapa que la identificamos con Participantes, la segunda, tercera y cuarta etapa las incluimos en nuestro Procedimiento que hemos seguido y la última etapa la identificamos como Evaluación; en cada una de estas, se identifican las acciones seguidas.

\section{Participantes}

Etapa I. Se identifica el problema de investigación en lo relacionado con la formación del docente en EEF. La muestra objeto de estudio fueron docentes de Matemática y 
Ciencias Sociales de 13 instituciones educativas del departamento del Atlántico, de las cuales II pertenecen a sectores oficiales y 2 a sectores privados, entre ellas uno bilingüe.

\section{Procedimiento}

Etapa 2. Para la recolección y el tratamiento de la información, se recurrió a entrevistas, observación y revisión documental (siguiendo los cuatro pasos de Quintana [2006]).A partir de estas, se logró:

- Rastrear e inventariar los documentos existentes: en lo relativo a EEF a nivel internacional y nacional, y de las instituciones educativas participantes sus planes de clase y mallas curriculares.

- Clasificar y seleccionar los documentos identificados.

- Leer en profundidad el contenido de los documentos seleccionados para extraer elementos de análisis.

- Leer en forma cruzada y comparativa los documentos en cuestión.

Etapa 3. Se establece el procedimiento de categorización y análisis de la caracterización de la investigación y la formación de los docentes en EEF. Con la triangulación de la información y convergencia, se analiza e interpretan los resultados obtenidos desde la revisión de documentos, observación y entrevistas. Y según estos soportes y hallazgos se elabora un plan de trabajo, el cual consistió en diseñar el programa de EEF mediado por tecnología para la formación del docente de Matemáticas y de Ciencias Sociales, para desarrollar competencias en EEF y a su vez brindar orientaciones, estrategias y herramientas para la planeación y ejecución de su enseñanza.

Etapa 4. Se establece la ejecución del plan de formación diseñado. Por medio de un curso gratuito con 120 horas en la modalidad B-learning dirigido a las 13 instituciones educativas, apoyado de una página web, diseñada exclusivamente para los propósitos en los que se consolidaron las orientaciones para el docente por medio de cuatro módulos de formación, seguido de actividades para estudiantes, se diseñó plan de seguimiento y acompañamiento por medio de la plataforma Classrom para el desarrollo en la parte virtual.

\section{Evaluación}

Etapa 5. Para esta parte del proceso, se elaboraron dos cuestionarios, inicial y de monitoreo, validados por juicios de expertos para luego ser aplicados. El cuestionario inicial constaba de seis preguntas abiertas que pretendían identificar los conocimientos en EEF en los docentes, su formación en este saber y su enseñanza, en atención a lo establecido por el MinEducación sobre la EEF. EI cuestionario de monitoreo comprendió seis preguntas abiertas realizadas por medio de la plataforma Classrom para examinar la incorporación de habilidades en EEF y herramientas tecnológicas en los docentes durante $y$ al finalizar el desarrollo del programa, a partir de las orientaciones brindadas y con estas hacer una evaluación del programa diseñado e implementado con los profesores de las instituciones educativas participantes del estudio.

De acuerdo con la información obtenida y con las teorías que soportan la investigación, se redactan reportes que se utilizan para evaluar la aplicación del programa llevando a cabo los ajustes necesarios para redefinir el problema y retroalimentar a los participantes.

\section{Resultados}

La identificación de las necesidades se hizo con la observación a docentes, entrevistas de opinión y escritas, lo cual dejó en evidencia el desconocimiento que tienen los docentes sobre los lineamientos en EEF del MinEducación, planteado como proyecto que atraviesa por todo el currículo desde las diferentes asignaturas. Ellos manifestaron que este saber es importante, ya que ayudaría a sus estudiantes a tomar buenas decisiones y tener un buen manejo y control de sus finanzas, lo cual les serviría para mejorar la calidad de vida. Por otro lado, no han recibido ningún tipo de formación o capacitación en esta área, sin embargo, algunos revelaron que se capacitan de forma autodidacta, puesto que las instituciones educativas donde laboran no consideran la EEF como lo plantea en MinEducación.

Para el diseño del programa de formación docente, se consideró lo descrito por Taborga y Hanel (1992) y Reyes (2002) en el sentido de dar operatividad con la aplicación de criterios importantes, ayudar al desarrollo de capacidades y competencias, $y$ articular acciones rutinarias e innovadoras.

Los hallazgos desde la revisión de documentos realizada en las 13 instituciones educativas de sectores oficiales $y$ privados de departamento del Atlántico reveló que no involucran la EEF en sus planes de área y mallas curriculares, a excepción de una institución, la cual promueve la EEF dentro de la institución desde los grado I-II con unas cartillas publicadas por Los Tres Editores. Estas cartillas involucran todos los lineamientos del MinEducación, se 
contemplan contenidos económicos y financieros, preguntas tipo Icfes (Instituto Colombiano para la Evaluación de la Educación) y competencias en EEF. En general, estas instituciones educativas desconocen el proyecto Mi plan, mi vida, mi futuro: orientaciones pedagógicas para la educación económica y financiera, cartillas y material de apoyo diseñado por el MinEducación sobre la EEF.

Por otro lado, se hizo la lectura cruzada de documentos que promueven la EEF a nivel nacional e internacional, $y$ se extrajeron las dimensiones de estrategias didácticas, contenidos y competencias, como se muestra en la Tabla I, aspectos que fueron considerados en el diseño del plan de formación docente.

El recurso educativo digital (RED), diseñado y utilizado para la organización y ejecución del programa de formación docente, fue creado bajo la licencia Creative Commons. Es alojado en eef-ua.herokuapp.com, es gratuito y accesible para todo público, y se llama Aprendamos educación económica y financiera en la matemática. También cuenta con orientaciones pedagógicas para el docente y el desarrollo de las actividades dirigidas a estudiantes (Figura 2).

Tabla I. Revisión documental guías, módulos y cartillas

\begin{tabular}{|c|c|c|c|}
\hline \multirow[t]{2}{*}{ Documentos, guías y cartillas } & \multicolumn{3}{|c|}{ Elementos para el diseño del programa } \\
\hline & Estrategias didácticas & Contenidos & Competencias \\
\hline $\begin{array}{l}\text { Mi plan, mi vida, mi futuro: orientaciones pedagógicas para la edu- } \\
\text { cación económica y financiera (MinEducación, }(2014)^{*}\end{array}$ & $\mathrm{X}$ & $\mathrm{X}$ & $\mathrm{x}$ \\
\hline Portal web Pesos Pensados junto con la ENEEF (CIEEF, 20I7)* & $x$ & $x$ & \\
\hline $\begin{array}{l}\text { Cartillas, fichas didácticas y portal web Saber Más ser Más, } \\
\text { Asobancaria en convenio con MinEducación }(2016)^{*}\end{array}$ & $\mathrm{x}$ & $\mathrm{X}$ & \\
\hline $\begin{array}{l}\text { Estándares básicos de competencias en matemáticas (MinEducación, } \\
\text { 1998)* }\end{array}$ & & & $\mathrm{x}$ \\
\hline Derechos básicos de aprendizaje: matemáticas (Min Educación, 2016)* & $\mathrm{x}$ & $\mathrm{X}$ & \\
\hline $\begin{array}{l}\text { Cuadernillo de prueba: ejemplo de preguntas Saber } 9^{\circ} \text { Educación } \\
\text { Económica y Financiera (MinEducación, 20I5)* }\end{array}$ & $\mathrm{X}$ & & \\
\hline $\begin{array}{l}\text { Manual para la educación no formal manual para facilitadores del Pro- } \\
\text { grama de Educación Social y Financiera de Aflatoun (Aflatoun, 2006)** }\end{array}$ & $\mathrm{X}$ & $\mathrm{X}$ & \\
\hline Manual Aflateen I(Aflateen, 2008)** & $x$ & $x$ & \\
\hline $\begin{array}{l}\text { Educación financiera en enseñanza secundaria obligatoria nivel l: } \\
\text { guía para el profesorado (Banco de España, 2009)** }\end{array}$ & $\mathrm{x}$ & $x$ & \\
\hline
\end{tabular}

*Documentos analizados a nivel nacional

**Documentos analizados a nivel internacional

Figura 2. Aprendamos educación económica y financiera en la matemática.

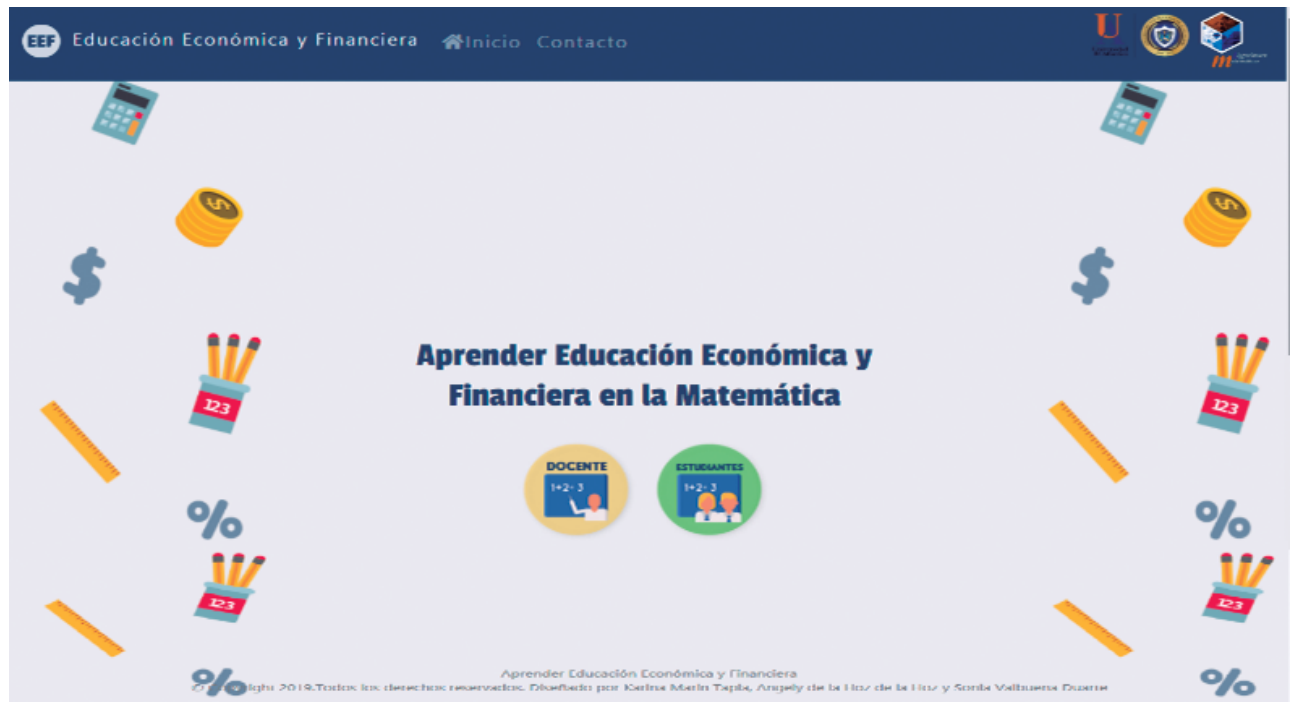


En el RED se crearon cuatro módulos de formación, los cuales cuentan con objetivos, desarrollo de contenidos económicos y financieros, estrategias didácticas, competencias en EEF. En el espacio del docente, se encuentra el objetivo del programa y los cuatro módulos de formación; cada módulo cuenta con la descripción de este y el contenido descargable en el que se halla la planeación de las actividades brindadas en un archivo PDF (Figura 3).

La Figura 4 ilustra el espacio del estudiante, constituido por el objetivo, los cuatro módulos de aprendizaje y un espacio en el que se le ha colocado material al estudiante, para que por medio de un archivo PDF pueda tener disponible los recursos que utilizará para el desarrollo de algunas actividades.

La estructura del programa de formación docente fue diseñada con el aporte de la lectura cruzada de la literatura de los estamentos internacionales y el Estado colombiano sobre EEF, en los que se extrajeron elementos para la planeación de cada módulo, como asignatura, grado escolar, módulo del aprendizaje y título del objeto de aprendizaje, y se obtuvieron cuatro categorías en el momento de planificar una clase, estas son:

- Objetivo de aprendizaje, enfocado en los conocimientos, las habilidades o las aptitudes que los estudiantes deben desarrollar como resultado de aprendizaje; en este sentido, las competencias en EEF.

- Habilidad/conocimiento, referida a la capacidad adquirida por los estudiantes durante el desarrollo del módulo de aprendizaje.

- Flujo de aprendizaje, representa la forma en que se va a transmitir el conocimiento, este se lleva a cabo mediante tres etapas: inicio, desarrollo y cierre.

- Guía de valoración, expone los aspectos a evaluar como producto de los aprendizajes, lo que los estudiantes comprenden, saben y pueden hacer (Figura 5).

Figura 3. Espacio diseñado para orientar al docente

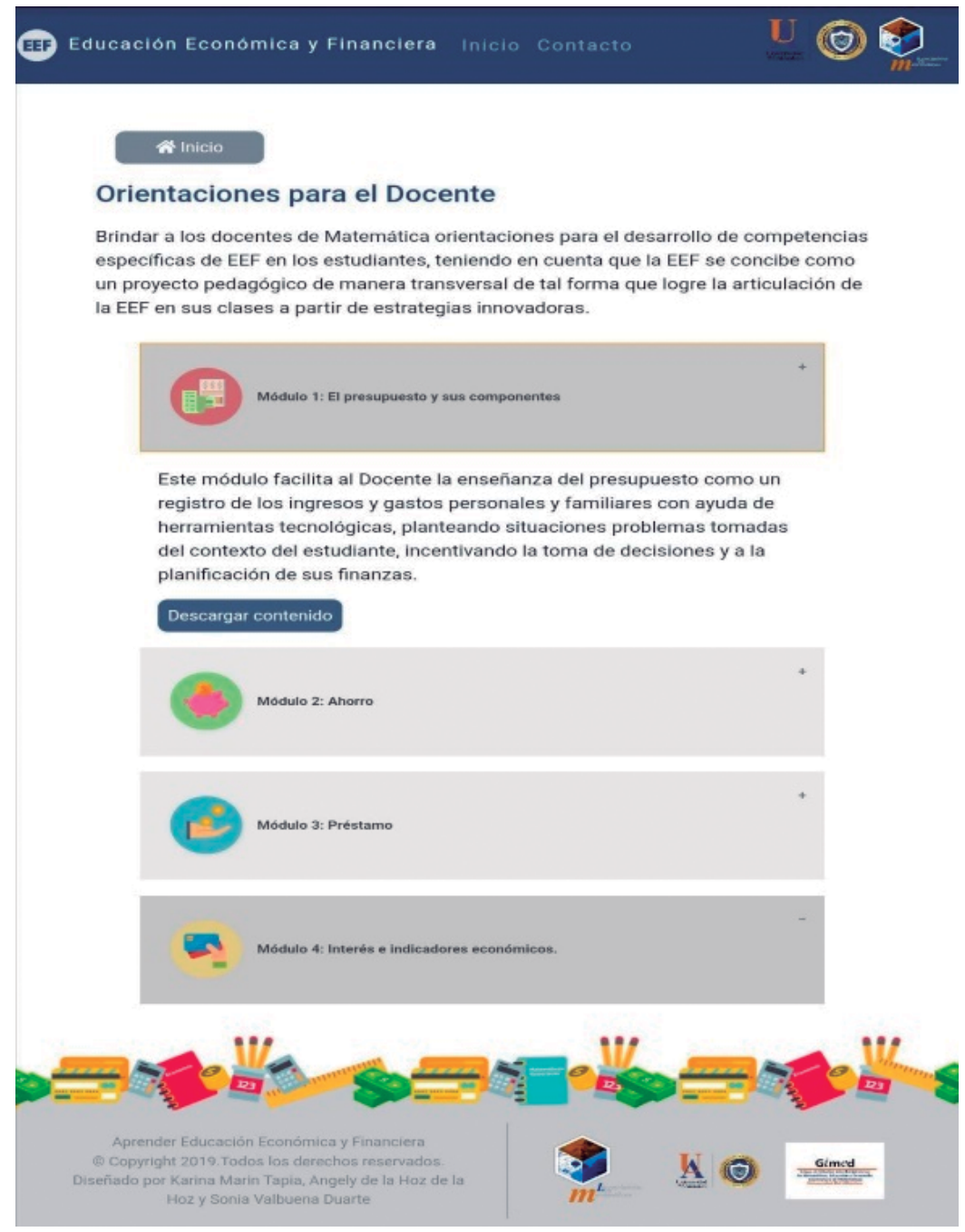


Figura 4. Espacio diseñado para el desarrollo de actividades en estudiantes.

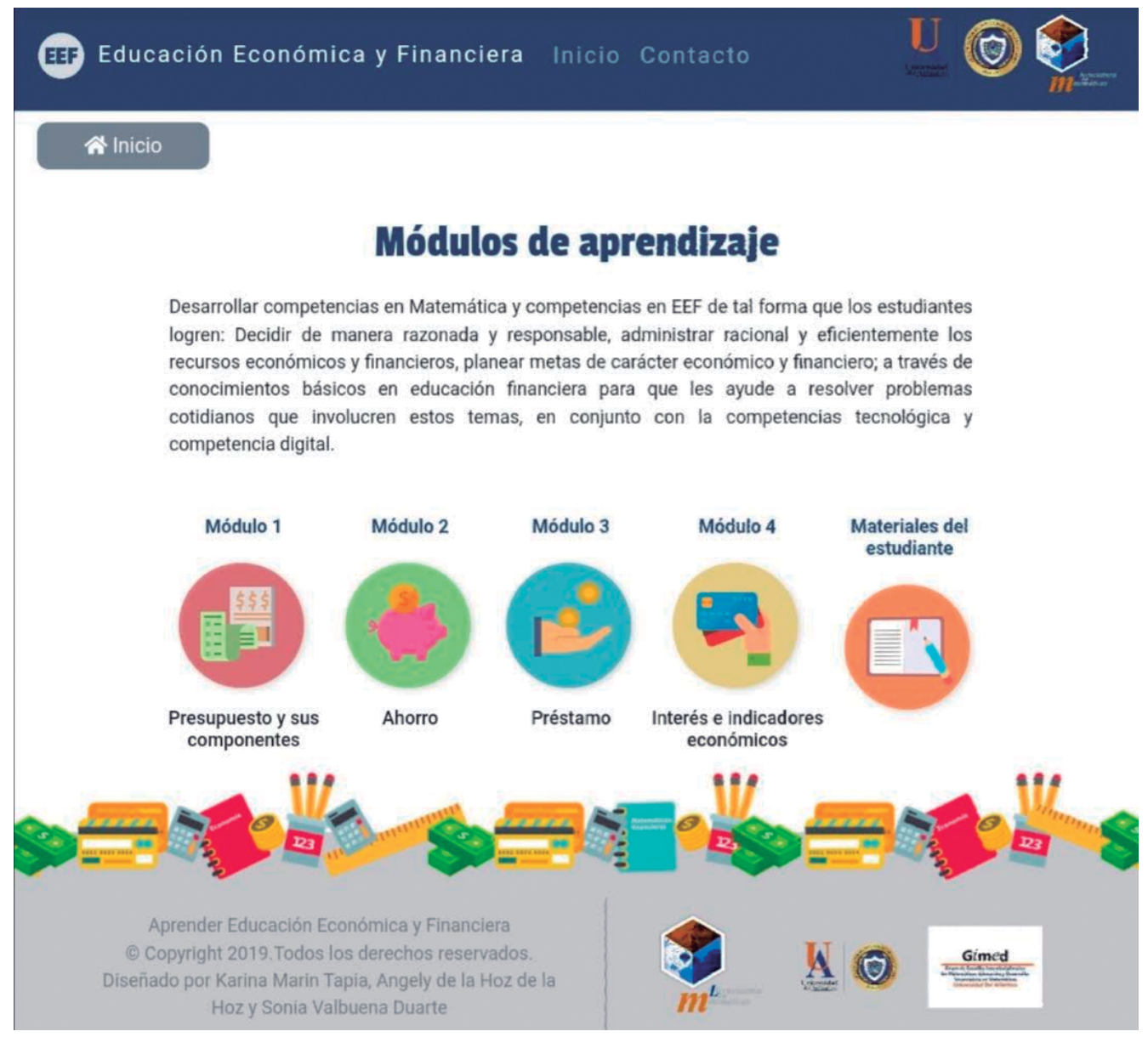

En cada módulo de formación el flujo de aprendizaje se llevó a cabo por medio de tres tipos de actividades: las de inicio, actividades de desarrollo y actividades de cierre.

Para el diseño de las actividades se siguió a Godino (2004), en la planeación y diseño de secuencias didácticas estableciendo las unidades didácticas de aprendizaje, considerando:

- Preguntas para iniciar la reflexión.

- Organización de la actividad, gestión, recursos, etc.

- Los contenidos y su organización.

- Actividades para continuar con la secuencia.

Las actividades de inicio propuestas en el programa fueron elaboradas en la plataforma Kahoot! con la intención de explorar los conocimientos previos de sus estudiantes por medio de preguntas de introducción. Para el desarrollo de los contenidos incluidos en las actividades, se diseñaron animaciones explicativas realizadas mediante la herramienta Sparkol Videoscribe, que permitieron abordar los contenidos temáticos y ponerlos a disposición de los estudiantes en la página web; para esto fue necesario utilizar la plataforma de YouTube (Figura 6).

Las actividades de desarrollo fueron diseñadas con ayuda de herramientas tecnológicas que permitieron captar la atención de los docentes y estudiantes, y propiciar un ambiente de aprendizaje innovador, con uso de aplicaciones y plataformas gratuitas como Educaplay, Genially, GeoGebra, Excel y formularios de Google (Figura 7).

La actividad de cierre fue diseñada por medio de un formulario de Google en el que se planteaban a los estudiantes un cuestionario con preguntas tipo Icfes de selección múltiple con única respuesta, el cual permitió evaluar los conocimientos aprendidos de los estudiantes durante el desarrollo del módulo y así avanzar en la complejidad de los contenidos de los demás módulos de aprendizaje.

La ejecución o el desarrollo del programa se realizó a través de un curso ofrecido por el Grupo de Investigación Estudios Interdisciplinarios en Matemáticas, Educación y 
Figura 5. Planeación de las actividades.

\begin{tabular}{|c|c|}
\hline $\begin{array}{l}\text { Asignatura } \\
\text { Matemáticas }\end{array}$ & $\begin{array}{c}\text { Módulo de aprendizaje } \\
\text { El presupuesto y sus } \\
\text { componentes }\end{array}$ \\
\hline \multicolumn{2}{|r|}{ Resuelve problemas utilizando las propiedades de números reales. } \\
\hline $\begin{array}{l}\text { Objetivos de } \\
\text { aprendizaje }\end{array}$ & $\begin{array}{l}\text { 1. Decidir de manera razonada y responsable que acciones desde el punto } \\
\text { de vista económico y financiero son pertinentes para su bienestar persona } \\
\text { y el de la comunidad. } \\
\text { 2. Interpretar situaciones problemas reconociendo que las personas tienen } \\
\text { recursos financieros limitados, haciendo uso de la toma de decisiones } \\
\text { informadas. } \\
\text { 3. Calcular el presupuesto resolviendo operaciones con números reales. } \\
\text { 4. Reconocer el presupuesto personal y familiar como una herramienta úti } \\
\text { para ayudarnos a planear y administrar nuestro dinero. } \\
\text { 5. Calcular porcentajes con variables relacionadas al IVA. }\end{array}$ \\
\hline $\begin{array}{l}\text { Habilidad/ } \\
\text { conocimiento }\end{array}$ & $\begin{array}{l}\text { 1. Identifica los componentes de un presupuesto. } \\
\text { 2. Diferencia los tipos de ingresos y gastos en un presupuesto. } \\
\text { 3. Reconoce las propiedades de los números reales, resolviendo operaciones } \\
\text { entre ellos. } \\
\text { 4. Utiliza herramientas tecnológicas para la resolución de problemas y } \\
\text { ejecución de actividades. } \\
\text { 5. Reconoce que las necesidades están por encima de los deseos. } \\
\text { 6. Interpreta situaciones problemas relacionado a un contexto. } \\
\text { 7. Calcula el IVA incluido en productos de consumo. }\end{array}$ \\
\hline $\begin{array}{c}\text { Flujo de } \\
\text { aprendizaje }\end{array}$ & $\begin{array}{l}\text { Inicio } \rightarrow \text { Desarrollo } \rightarrow \text { Cierre } \\
\text { Inicio: Preguntas de introducción } \\
\text { Desarrollo } \\
\text { Actividad 1: Mis necesidades y deseos } \\
\text { Actividad 2: Elaborando presupuesto personal y familiar } \\
\text { Actividad 3: Conociendo los ingresos y gastos de la Nación } \\
\text { Cierre: Cuestionario de preguntas relacionado a temas vistos en las } \\
\text { actividades. }\end{array}$ \\
\hline $\begin{array}{l}\text { Guía de } \\
\text { valoración }\end{array}$ & $\begin{array}{l}\text { Se espera que los estudiantes analicen situaciones relacionadas a un } \\
\text { contexto, identificando la toma de decisiones informadas y además } \\
\text { resuelvan operaciones con números reales al momento de calcular un } \\
\text { presupuesto. } \\
\text { Adicionalmente, en esta actividad se debe evaluar la habilidad comunicativa } \\
\text { de los estudiantes a través de la forma en que expresen sus ideas. }\end{array}$ \\
\hline
\end{tabular}


Figura 6. Interfaz de la temática en EEF diseñada con la aplicación SparkolVideoscribe

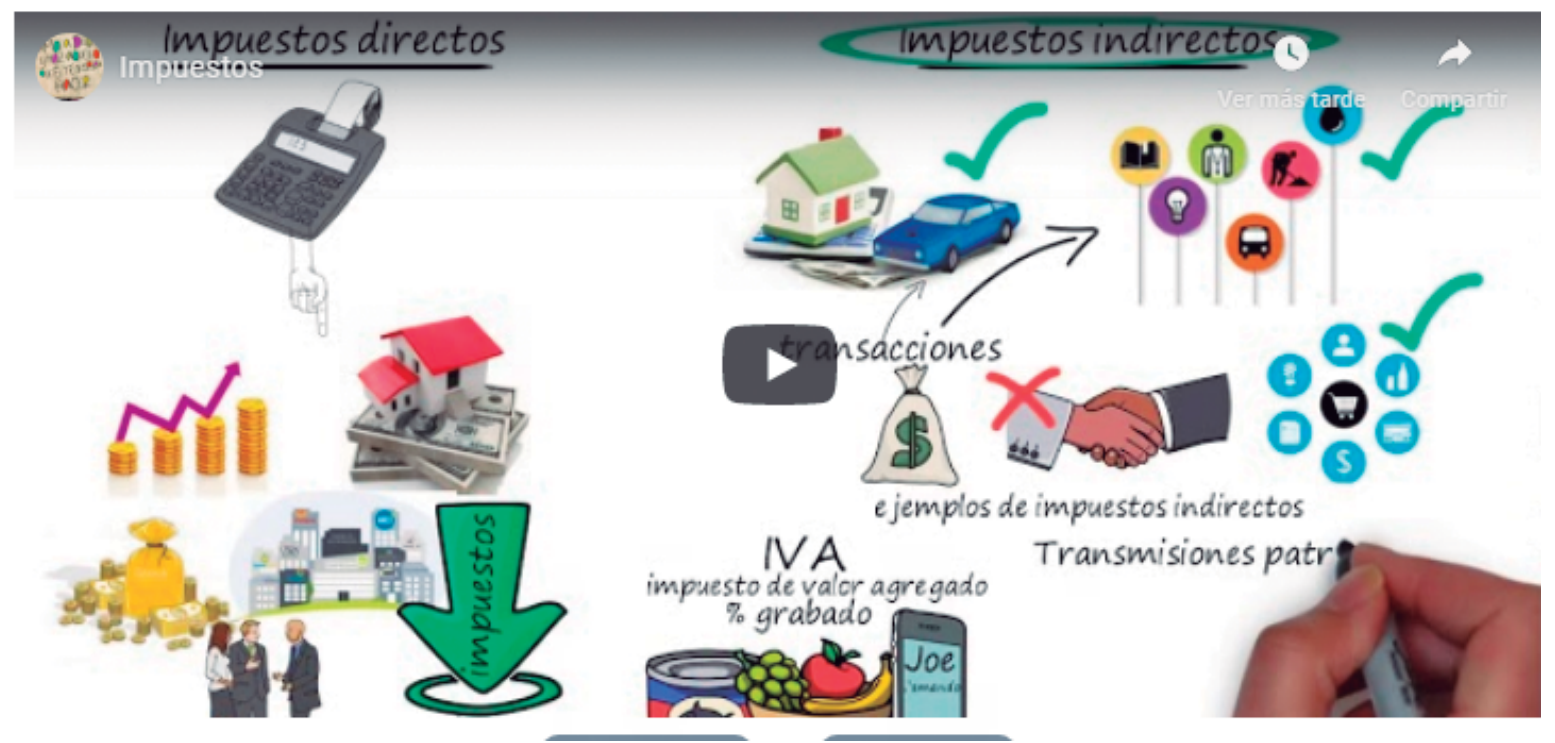

Figura 7. Herramientas tecnológicas utilizadas para diseño el de actividades.

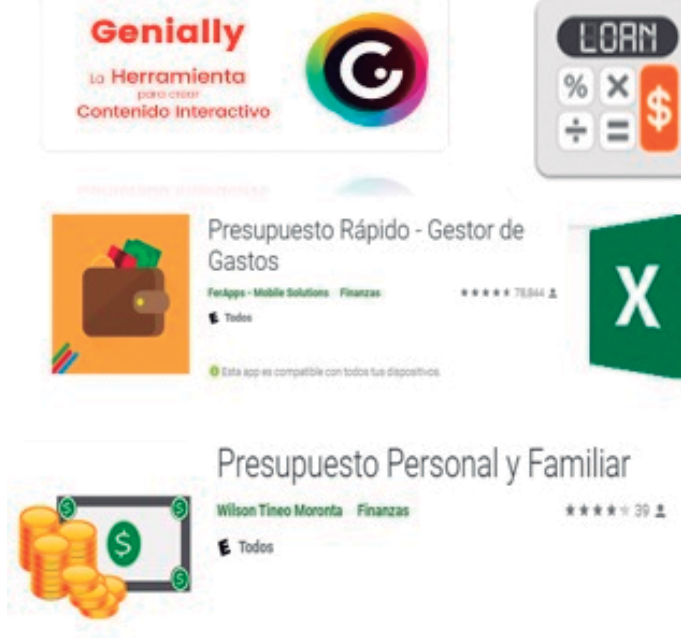

Desarrollo (GIMED) de la Universidad del Atlántico, en la modalidad b-learning, convocando a 13 instituciones educativas del departamento del Atlántico. El curso se desarrolló en 120 horas (Tabla 2), se hizo contacto presencial con los docentes y se usaron espacios universitarios y espacios de los colegios.

Tabla 2. Desarrollo de módulos por semana

\section{Módulos de formación}

Módulo I

El presupuesto y sus componentes

Módulo 2

Ahorro

Módulo 3

Préstamo

Módulo 4

\section{Modalidad}

Presencial

Presencial/virtual

Presencial/virtual

Virtual

\section{\&. GeoGebra \\ soffware Dinámico de Matemásicas}
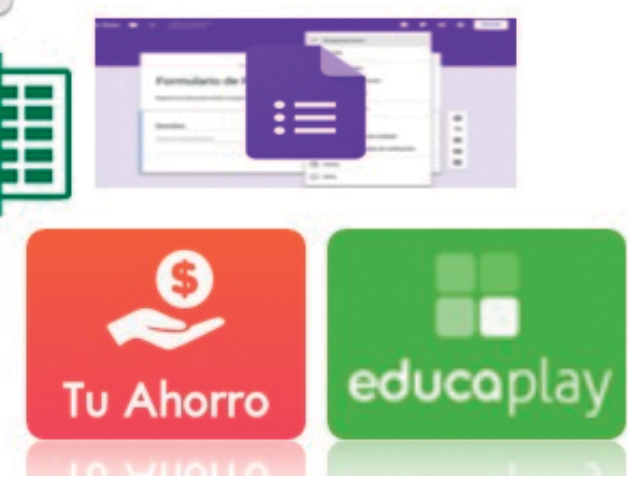

Para el trabajo en la modalidad virtual, se usó el sitio web diseñado en especial para la ejecución del programa, el cual es un recurso educativo digital de acceso abierto y se encuentra disponible para toda la comunidad educativa. A su vez, se hizo seguimiento y acompañamiento por medio de la plataforma Classrom y se utilizó un foro de preguntas para compartir la experiencia, $y$ en el cual los docentes valoraron el programa de formación realizado.

\section{Discusión y conclusiones}

El programa de formación docente se diseñó identificando y extrayendo elementos que lo constituyen. De esta manera, se conformaron las orientaciones con la planeación 
de los módulos de formación y se consolidó en forma directa en un recurso educativo digital abierto.

Los indicadores de validez para la evaluación del programa fueron identificados en la construcción teórica, en consideración a la evaluación como parte del conjunto de criterios de calidad orientado por principios de consistencia y credibilidad.

El programa se sometió a validación por pilotaje formando a un grupo de docentes de Matemáticas y Ciencias Sociales de 13 instituciones del departamento del Atlántico (Colombia), en consideración a que el desarrollo de los contenidos, las estrategias didácticas y el uso de herramientas tecnológicas aportan al desarrollo de competencias en EEF y permiten su desarrollo en los estudiantes.

Con el producto tangible de la investigación, se logró impactar en EEF a los docentes de Matemáticas y Ciencias Sociales participantes, quienes aprendieron a construir actividades con el uso de herramientas tecnológicas y reconocieron que las orientaciones brindadas en cada uno de los módulos permiten desarrollar de una forma dinámica competencias en EEF en sus estudiantes y que además la estructura en que se desarrollan los módulos es un ejemplo a seguir para enriquecer su práctica laboral.

La evaluación del programa fue producto del análisis del cuestionario de monitoreo, de las experiencias y de las opiniones de los docentes acerca de las orientaciones brindadas. Estos mostraron una actitud positiva con respecto a la planeación de cada uno de los módulos de formación, la estructura de las actividades y los recursos utilizados para su desarrollo.

El recurso educativo digital (RED) es abierto, por lo que puede tener aportes de la comunidad que incrementen la formación en las competencias en educación económica y financiera para la toma de decisiones informadas del ciudadano común, y podrían considerarse elementos y aportes que contribuyan en estas competencias a través de tener una visión general del Sistema financiero en Colombia (Zuleta, 1997) y en aspectos relativos a liberalización, inversión extranjera, Cartera, tasas de interés, margen de intermediación, entre otros.

\section{Referencias}

Aflateen, M. (2008). Manual Aflateen I. Recuperado de https:// docplayer.es/ I3386305-Aflateen-manual-manual-aflateen.html
Aflatoun. (2006). Manual para la educación no formal manual para facilitadores del Programa de Educación Social y Financiera de Aflatoun. Recuperado de https://es.calameo.com/ $\mathrm{read} / 003436347835 \mathrm{blbf62027}$

Asociación Bancaria y de Entidades Financieras de Colombia. (2016). Experiencias y aprendizajes de la educación financiera. Recuperado de http://www.asobancaria.com/ sabermassermas/wp-content/uploads/2016/08/CartillaEducacion-Financiera-Asobancaria-Mayo-Sin-lineas-deImpresion.pdf

Banco de España. (2009). Educación financiera en enseñanza secundaria obligatoria nivel l: guía para el profesorado. Madrid, España:Autor.

Comisión Intersectorial para la Educación Económica y Financiera. (2017). Estrategia Nacional de Educación Económica y Financiera. Recuperado de https://www.banrep. gov.co/es/publicaciones/estrategia-nacional-educacioneconomica-financiera

Creswell, J. (20I2). Educational research planing, conducting and evaluating quantitative and qualitative research. (4. ${ }^{\mathrm{a}}$ ed.). Boston, EE. UU.: Pearson.

Cristancho Prada, M. M., Cruz,Y. X., Echeverry,Y.P., Rojas, D. R., \& Urrego Bedoya, M. E. (20I7). Educación financiera para estudiantes de media vocacional del Colegio Pablo Neruda en Bogotá, DC (Tesis de grado, Corporación Universitaria Minuto de Dios, Bogotá, Colombia). Recuperado de https:// repository.uniminuto.edu/handle// 0656/5884

Domínguez Martínez, J. M. (2015). La educación financiera: inventario de cuestiones planteadas. eXtoikos, I7, 3-7. Recuperado de https://dialnet.unirioja.es/servlet/ articulo?codigo $=5555386$

El Heraldo. (2013, Junio 26). En Barranquilla, comienza programa nacional de educación financiera. Recuperado de https://www.elheraldo.co/noticias/economia/en-barranquilla-comienza-programa-nacional-de-educacion-financiera- II5293

Gamboa Peña, M. O., Hernández Suárez, C. A., \& Avendaño Castro, W. R. (20I8). La importancia de la educación financiera para niños en edad escolar. Revista Espacios, 40(2), 9. Recuperado de https://www.revistaespacios. com/a 19v40n02/a 19v40n02p06.pdf

Godino, J. (Dir.) (2004). Didáctica de las matemáticas para maestros. Granada, España: Universidad de Granada.

Hernández Sampieri, R., Fernández Collado, C., \& Baptista Lucio, P. (20I4). Metodología de la investigación. (6. ${ }^{a}$ ed.). Ciudad de México, México: McGraw-Hill. 
Hernández Madroñero, I., Londoño Cardozo, J., \& Silva Mazo, L. M. (20/9). El pensamiento crítico y sus beneficios en la Administración. Revista Logos Ciencia \& Tecnología, I I (I), 6I-76. http://dx.doi.org//0.22335/rlct.vI lil.694

Instituto Superior de Educación Rural. (2015). Plan de formación y capacitación docente 2015-2020. Recuperado de http://www.iser.edu.co/iser/hermesoft/portallG/ home_l/recursos/documentos_generales/290720I5/ plan_forma_capac_doc_2015_2020.pdf

Ley 1328/2009, de 15 de julio, por la cual se dictan normas en materia financiera, de seguros, del mercado de valores y otras disposiciones. Diario Oficial, núm. 474II (2009).

Ministerio de Educación Nacional. (1998). Estándares básicos de competencias en matemáticas. Recuperado de http:// www.mineducacion.gov.co/cvn/I665/articles-I 16042_archivo_pdf2.pdf

Ministerio de Educación Nacional. (20l4). Mi plan, mi vida, mi futuro: orientaciones pedagógicas para la educación económica y financiera. Bogotá, Colombia: Autor. Recuperado de https://www.mineducacion.gov.co//759/articles-343482_archivo_pdf_Orientaciones_Pedag_Educ_ Economica_y_Financiera.pdf

Ministerio de Educación Nacional. (20I5). Cuadernillo de prueba: ejemplo de preguntas Saber $9^{\circ}$ Educación Económica y Financiera. Bogotá, Colombia:Autor.

Ministerio de Educación Nacional. (2016) Derechos básicos de aprendizaje: matemáticas. Bogotá, Colombia:Autor.

Ministerio de Hacienda y Crédito Público. (2014). decreto 457 del 2014. Bogota. Recuperado de http://bancadelasoportunidades.gov.co/sites/default/files/2017-.pdf

Mogollón-Guerrero, M. A., Molano-Castro, M. Z., \& Mesa-Jiménez, F.Y. (20I7). Una mirada a la educación económica y financiera en Básica Primaria. Pensamiento y Acción, 23, 49-62. Recuperado de https://revistas.uptc.edu.co/index. php/pensamiento_accion/article/view/8446

Murillo Junco, M. J., \& Cáceres Castellanos, G. (2013). Business intelligence y la toma de decisiones financieras: una aproximación teórica. Revista Logos, Ciencia \& Tecnología, 5(I), I 19-138. https://doi.org//0.22335/RLCT.V5II.| |

Organización para la Cooperación y el Desarrollo Económicos. (2005). Principles and good practices for financial education and awareness. Recuperado de http://www.financialstabilityboard.org/2005/06/cos_050622/
Organización para la Cooperación y el Desarrollo Económico. (2007). El programa PISA de la OCDE: qué es y para qué sirve. Recuperado de https://www.oecd.org/pisa/publicacionesdepisaenespaol.htm

Organización para la Cooperación y el Desarrollo Económico. (20I4). Resultados de PISA 2012 en foco: Io que los alumnos saben a los 15 años de edad y lo que pueden hacer con lo que saben. Recuperado de https://www.oecd.org/pisa/ keyfindings/PISA2012_Overview_ESP-FINAL.pdf

Quintana Peña, A. (2006). Metodología de investigación científica cualitativa. En A. Quintana Peña, \& W. Montgomery (Eds.), Psicología tópicos de actualidad. (Pp. 65-73). Lima, Perú: Universidad Nacional Mayor de San Marcos. Recuperado de http://biblioteca.udgvirtual.udg.mx:8080/jspui/ handle/ I23456789/2724

Reyes Zúñiga, E. (2002). Los programas de formación docente: una visión más allá del aula. Revista Internacional de Ciencias Sociales y Humanidades, I2(2), 207-2 I8. Recuperado de https://www.redalyc.org/pdf/654/654I 22I I.pdf

Semana. (2018, enero 20). La resaca del endeudamiento. Recuperado de https://www.semana.com/economia/articulo/alto-endeudamiento-de-los-hogares-la-cruda-reali$\mathrm{dad} / 554143$

Sepúlveda Aravena, J. (2014). Educación económica y financiera para la formación inicial de profesores: herramientas conceptuales y didácticas. Temuco, Chile: Universidad de la Frontera. Recuperado de http://humanidades.ufro.cl/images/libros/Educacion_Economica_y_financieracompressed.pdf

Stufflebeam, D. L., \& Shinkfield,A.J. (1987). Evaluación sistemática: guía teórica y práctica. Barcelona, España: Paidós.

Taborga, H., \& Hanel, J. (1992). Elementos para la evaluación del sistema de educación superior en México. Revista de la Educación Superior, 82.

Universidad de Colima. (20/2). Programa Institucional de Formación Docente. Dirección general de desarrollo del personal académico. Recuperado de https://portal.ucol. $\mathrm{mx} /$ content/micrositios/I I8/file/PIFOD.pdf

Zuleta, H. (1997). Una visión general del sistema financiero colombiano. Revista del Banco de la República, 70(835), 1942. Recuperado de https://publicaciones.banrepcultural. org/index.php/banrep/article/view/II 765 